\title{
Quantum nonlocality in the presence of superselection rules and data hiding protocols
}

\author{
F. Verstraete and J.I. Cirac \\ Max-Planck-Institut für Quantenoptik, Hans-Kopfermann-Str. 1, Garching, D-85748, Germany.
}

(Dated: February 5, 2003)

\begin{abstract}
We consider a quantum system subject to superselection rules, for which certain restrictions apply to the quantum operations that can be implemented. It is shown how the notion of quantumnonlocality has to be redefined in the presence of superselection rules: there exist separable states that cannot be prepared locally and exhibit some form of nonlocality. Moreover, the notion of local distinguishability in the presence of classical communication has to be altered. This can be used to perform quantum information tasks that are otherwise impossible. In particular, this leads to the introduction of perfect quantum data hiding protocols, for which quantum communication (eventually in the form of a separable but nonlocal state) is needed to unlock the secret.
\end{abstract}

The laws of Quantum Mechanics allow us to carry certain tasks that would be impossible in a classical world. The strength of quantum information theory resides in a subtle interplay between the grandeur of the Hilbert space and the limitations on the allowed operations. One of the most prominent examples is quantum cryptography [1], where the existence of quantum superpositions or entangled states on one hand and the limitations due to the no-cloning theorem [2] on the other hand ensure the possibility of a secure transmission of information between two or more partners.

The power of quantum mechanics becomes especially apparent when natural limitations apply to the operations that one can apply. As an example, the theory of entanglement and quantum nonlocality arises from the restriction to local operations and classical communication (LOCC). It is somehow expected that additional constraints would lead to new interesting physics and applications, especially in the context of cryptography.

In many physical systems of interest, such an additional restriction applies in form of superselection rules: an extra axiom of quantum mechanics dictates the existence of superselection rules forbidding e.g. coherent superpositions between states of elementary particles with different electric charge [3, 4]. In practice, energy constraints lead to effective superselection rules. We will consider a particular instance of a superselection rule, which is deeply motivated by current quantum optical experiments, namely particle number. This corresponds, for example, to the situation in which one has bosonic atoms as it is the case in experiments with cold atomic gases [5]. In this case, all physical observables (and hence states) commute with the particle number operator. Our results however also apply other types of superselection rules.

In this paper we will show that in the presence of superselection rules it is possible to carry out tasks which otherwise cannot be performed in the context of quantum information. The underlying cause appears to be that superselection rules completely alter the notion of local distinguishability of quantum states: it is not true anymore that any two pure orthogonal states can be distinguished using LOCC, as is always possible without superselection rules [6]. We will exploit this fact to construct a data hiding protocol 7, 8], where the goal is to distribute some information (classical or quantum) among several partners in such a way that they can only "read" it if they are provided with the means to perform joint measurements. It has been proven that a perfect scheme is impossible [7]. In the presence of superselection rules however, we will show that perfect data hiding is possible, and that one can do it with pure states.

Furthermore, we will show that our view of entanglement and quantum nonlocality has to be altered 9, 10, 11]. Entangled states are usually defined as those which cannot be prepared by LOCC out of a product state. This definition highlights the fact that in order to entangle two systems they must interact with each other (eventually via some third system). It distinguishes these states from those that are classically correlated (equivalently, separable), i.e., which can be written in the form 12

$$
\rho=\sum_{k} p_{k}\left|a_{k}\right\rangle\left\langle a_{k}|\otimes| b_{k}\right\rangle\left\langle b_{k}\right|
$$

where $1 \geq p_{k} \geq 0$ and $\left|a_{k}\right\rangle$ and $\left|b_{k}\right\rangle$ are normalized states. When we are dealing with superselection rules however, it happens that separable states cannot necessarily be prepared locally anymore, giving rise to the existence of states that are separable but nonlocal. We will show how this gives rise to a new type of nonlocal resource, as these states can be used to act as mediators to implement operations that are impossible by LOCC.

Finally, we will also show that all quantum information protocols can also be implemented in the presence of such superselection, so that these rules in practice do not impose any restriction to what one can do in practice, but rather offer novel ways to implement protocols that otherwise may not be possible. Note however that, unfortunately, the impossibility proof of quantum bit commitment [13] remains valid 14].

We start out considering a set of particles and the corresponding Hilbert space $H$. We can always decompose 


$$
H=\oplus_{N=0}^{\infty} H_{N}
$$

where $H_{N}$ is a subspace with a total number $N$ of particles. We assume that the particle number is a superselection observable, in the sense that the corresponding operator commutes with all observables [4]. This immediately imposes that superposition of pure states with different particle number cannot be prepared. This is the case, for example, in all the experiments with atoms or electrons. Any density operator must therefore admit a decomposition of the form

$$
\rho=\sum_{N=0}^{\infty} p_{N} \rho_{N}
$$

where $\rho_{N}$ is supported in $H_{N}$.

The situation becomes more intriguing when we consider two systems, A and B, spatially separated. Then, $H_{N}=\oplus_{n=1}^{N}\left(H_{n}^{A} \otimes H_{N-n}^{B}\right)$, where $H_{n}^{A}\left(H_{n}^{B}\right)$ denotes a Hilbert space corresponding to system A (B), with $n$ particles. The superselection rule combined with locality imposes that $A$ and $B$ cannot prepare superposition states of different local number of particles by LOCC. As we now show, this has very deep consequences in the concept of entanglement. In fact, there might be states which are separable but still they cannot be prepared locally, and therefore they are nonlocal. Let us consider two simple examples of such states. We take the simplest case in which $H^{A, B}$ are one-dimensional, i.e. they are spanned by the vectors $|n\rangle_{A, B}$ with $n$ particles.

Example 1:

$\rho_{1}=\frac{1}{4}\left(|0\rangle_{A}\langle 0|\otimes| 0\rangle_{B}\langle 0|+| 1\rangle_{A}\langle 1|\otimes| 1\rangle_{B}\langle 1|\right)+\frac{1}{2}\left|\Psi_{+}\right\rangle_{A B}\left\langle\Psi_{+}\right|$,

where $\left|\Psi_{+}\right\rangle_{A B}=\left(|0\rangle_{A}|1\rangle_{B}+|1\rangle_{A}|0\rangle_{B}\right) / \sqrt{2}$. This state is separable and has a very simple separable decomposition with $p_{k}=1 / 4(k=1,2,3,4)$ and

$$
\begin{aligned}
& \left|a_{1,2}\right\rangle=\left|b_{1,2}\right\rangle:=\frac{1}{\sqrt{2}}(|0\rangle \pm|1\rangle), \\
& \left|a_{3,4}\right\rangle=\left|b_{3,4}\right\rangle:=\frac{1}{\sqrt{2}}(|0\rangle \pm i|1\rangle) .
\end{aligned}
$$

Note that all these states are not compatible with the local version of the superselection rule, since they involve a superposition of different number of particles, and this applies to all separable decompositions.

Example 2:

$$
\rho_{2}=\int_{0}^{2 \pi} \frac{d \phi}{2 \pi}\left|\alpha e^{i \phi}\right\rangle_{A}\left\langle\alpha e^{i \phi}|\otimes| \alpha e^{i \phi}\right\rangle_{B}\left\langle\alpha e^{i \phi}\right|,
$$

where $\alpha>0$ and

$$
\left|\alpha e^{i \phi}\right\rangle:=e^{-\alpha^{2} / 2} \sum_{n=0}^{\infty} \frac{\alpha^{n}}{\sqrt{n !}} e^{i \phi n}|n\rangle,
$$

denotes a coherent state. This state can be written in the form (3) since it commutes with the total number operator, and therefore is compatible with the superselection rule. On the other hand, this state is obviously separable, though the states $\left|\alpha e^{i \phi}\right\rangle$ (17) are incompatible with the superselection rule.

Let us now show that the states (46) cannot be prepared locally if superselection rules apply. We will first derive a general result which is not only valid for the simple case in which $H_{n}^{A, B}$ are one-dimensional. Thus, these subspaces could now have an arbitrary number of dimensions, which include, for example, the use of ancilliary systems, and other modes or degrees of freedom of the particles. We just need to define $P_{n}^{A}$, the projector onto $H_{n}^{A}$ and analogously for $P_{n}^{B}$.

Proposition 1: If $\rho$ can be prepared locally, then

$$
\rho=\mathcal{N}(\rho):=\sum_{n_{A}, n_{B}=0}^{\infty}\left(P_{n_{A}}^{A} \otimes P_{n_{B}}^{B}\right) \rho\left(P_{n_{A}}^{A} \otimes P_{n_{B}}^{B}\right) .
$$

Proof: $\rho$ can be written as a convex combination of $\left|a_{n_{A}}\right\rangle_{A} \otimes\left|b_{n_{B}}\right\rangle$ with $\left|a_{n_{A}}\right\rangle \in H_{n_{A}}^{A}$ and $\left|b_{n_{B}}\right\rangle \in H_{n_{B}}^{B}$ which themselves fulfill (8). One can easily show that ancilliary systems do not affect this property.

Coming back to Examples 1 and 2, we have

$$
\begin{aligned}
\mathcal{N}\left(\rho_{1}\right) & =\frac{1}{4} \sum_{n, m=0}^{1}|n\rangle_{A}\langle n|\otimes| m\rangle_{A}\langle m| \neq \rho_{1}, \\
\mathcal{N}\left(\rho_{2}\right) & =\int_{0}^{2 \pi} \frac{d \phi_{1}}{2 \pi}\left|\alpha e^{i \phi_{1}}\right\rangle_{A}\left\langle\alpha e^{i \phi_{1}}\right| \\
& \otimes \int_{0}^{2 \pi} \frac{d \phi_{2}}{2 \pi}\left|\alpha e^{i \phi_{2}}\right\rangle_{B}\left\langle\alpha e^{i \phi_{2}}\right| \neq \rho_{2} .
\end{aligned}
$$

Thus, as announced above, both $\rho_{1,2}$ are separable states which cannot be locally prepared and are therefore expected to exhibit some kind of nonlocal properties.

The dual problem to the local preparation of quantum states is the problem of locally distinguishing quantum states (eventually with the help of classical communication). In the presence of superselection rules, the following applies:

Proposition 2: The states $\rho$ and $\mathcal{N}(\rho)$ cannot be distinguished using local operations and classical communication (LOCC).

Proof: First, note that we do not need to consider POVMs since we can always include the state of the ancillas in $\rho$. The operator corresponding to any observable $X_{A}\left(X_{B}\right)$ that Alice (Bob) measures has to commute with her (his) particle number operator, and thus we can write $\operatorname{tr}\left[\left(X_{A} \otimes X_{B}\right) \rho\right]=\operatorname{tr}\left[\mathcal{N}\left(X_{A} \otimes X_{B}\right) \rho\right]=$ $\operatorname{tr}\left[\left(X_{A} \otimes X_{B}\right) \mathcal{N}(\rho)\right]$.

Now, let us turn to introduce a quantum information task which makes use of these ideas. Since the local distinguishability is drastically affected by the presence of superselection rules, it is natural to investigate quantum 
data hiding protocols [7, 8]. We will show how a third party can give a secret bit to Alice and Bob which cannot be disclosed if they are only allowed to use local operations and classical communication (LOCC). If the bit is 0 or 1 , the state

$$
| \pm\rangle:=\frac{1}{\sqrt{2}}\left(|0\rangle_{1}|1\rangle_{2} \pm|1\rangle_{1}|0\rangle_{2}\right),
$$

is prepared, respectively. Then system 1 is given to Alice whereas system 2 to Bob. Note that the states $| \pm\rangle$ contain a superposition of two one-particle states and can therefore be prepared by the third party. In order to show that Alice and Bob can get no information about the bit, it is sufficient to note that $\mathcal{N}(|+\rangle\langle+|)=\mathcal{N}(|-\rangle\langle-|)$ : proposition 2 ensures that Alice and Bob cannot learn the value of the bit, even if they use classical communication. This value can be obviously read if they are allowed to perform joint operations. Notice that the scheme is perfect and uses pure states, in contrast to what happens in the scenario without superselection rules where perfect data hiding is not possible [7]. Note also that following 7], the present scheme can be used to hide quantum bits.

Next, we analyze the resources needed by Alice and Bob to learn the value of the bit if they can perform LOCC and they share entanglement. First, let us assume that they are given the entangled state

$$
|\Psi\rangle_{A B}=\frac{1}{\sqrt{N+1}} \sum_{n=0}^{N}|n\rangle_{A}|N-n\rangle_{B} .
$$

Thus, the total state will be

$$
\begin{aligned}
& \left|\Psi_{ \pm}\right\rangle \propto|0,0\rangle_{A}|1, N\rangle_{B} \pm|1, N\rangle_{A}|0,0\rangle_{B} \\
& \quad+\sum_{n=1}^{N}\left(|0, n\rangle_{A}|1, N-n\rangle_{B} \pm|1, n-1\rangle_{A}|0, N-n+1\rangle_{B}\right) .
\end{aligned}
$$

We consider the following local measurement. Alice and Bob measure both in the orthonormal basis composed of

$$
| \pm, n\rangle:=\frac{1}{\sqrt{2}}(|0, n\rangle \pm|1, n-1\rangle)
$$

$(n>0)$ and $|+, 0\rangle:=|0,0\rangle,|-, 0\rangle:=|1, N\rangle$. Then, each of them assigns the value 0 (1) to the measurement if the outcome of the measurement corresponded to one of the states $|+, n\rangle(|-, n\rangle)$ for some $n$. In case both assignments are the same (different), then they infer that the hidden bit was 0 (1). One can easily see that they will guess the value of the bit with a probability $N /(N+$ $1)$. We see that the probability is smaller than one but approaches this value for $N \rightarrow \infty$.

It seems to be a distinctive feature of distributed quantum systems subject to superselection rules that no perfect discrimination is possible in the presence of a bounded amount of nonlocal resources: unlike the usual case where teleportation can be used to create a quantum channel through a classical channel assisted by entanglement, this is in general not possible anymore in the presence of superselection rules. A classical channel assisted by a finite amount of entanglement is not equivalent anymore to a quantum channel [15]. In particular, this implies that our quantum data hiding scheme can be made arbitrary secure in the presence of a bounded amount of nonlocal resources shared between Alice and Bob [16].

Now we show that the separable states that cannot be prepared locally can also help to reveal the value of the hidden bit. This indeed proves that they are "more useful" to perform certain tasks than the ones that can be prepared locally, and therefore give rise to a new kind of nonlocal resource 15. We consider that, apart from the shared state, they are given the separable state $\rho_{2}$ [Eq.(6)]. Then, they perform the same local measurement as before and choose the value of the bit in the same way. Let us denote by $P_{x, y, n, m}^{z}$ the probability that they obtain the outcomes corresponding to $|x, n\rangle$ and $|y, m\rangle$, respectively, if the bit was $z(x, y= \pm$ and $z=0,1)$. We have

$$
\begin{aligned}
f_{n, m}(\alpha) & =P_{+,+, n, m}^{0}=P_{-,-, n, m}^{0}=P_{+,-, n, m}^{1}=P_{-,+, n, m}^{1} \\
& =\frac{e^{-2 \alpha^{2}}}{4} \frac{|\alpha|^{2(n+m-1)}}{n ! m !}|\sqrt{n}+\sqrt{m}|^{2} .
\end{aligned}
$$

One can readily prove that $\sum_{n, m=1}^{\infty} f_{n, m}(\alpha)$ tends to 1 in the limit $\alpha \rightarrow \infty$. Thus, the probability of detecting the value of the bit can be made arbitrarily close to one without using a non-separable state, although we have proven that this was not possible with LOCC operations.

Let us next formulate a few extensions to the present quantum data hiding scheme. First of all, it can easily be shown that Alice and Bob cannot extract any information even if they are given multiple copies of the same hiding state: this follows from the fact that $\mathcal{N}\left(\rho_{1}^{\otimes M}\right)=\mathcal{N}\left(\rho_{2}^{\otimes M}\right)$ for any number of copies $M$. This fact could be very useful when quantum data hiding would be implemented in a realistic environment with decoherence. Secondly, our scheme can readily be generalized to the multipartite setting [8]. Consider for example the following $N$-party state:

$$
| \pm\rangle: \propto|0\rangle_{1}|1\rangle_{2} \cdots|N-1\rangle_{N} \pm|1\rangle_{1}|2\rangle_{2} \cdots|N\rangle_{N-1}|0\rangle_{N}
$$

One immediately sees that this hiding scheme is perfectly secure, even when $N-1$ parties would decide to join forces. Note that more sophisticated versions can readily be constructed.

So far we have shown that the particle number superselection rules restricts the action that can be performed locally, which may be used to perform certain tasks that otherwise would be impossible. This seems to suggest that such rules may restrict some quantum information protocols. Now we show that this is not the case, i.e. 
that it is always possible to perform such protocols. The idea is quite simple and consists of noticing that we can always consider states that have a fixed number of local particles. Thus, if we want to have a protocol using a $d+1$-level systems in one particular location, we can just take states of the form $|n\rangle:=|n, d-n\rangle(n=0,1, \ldots, d)$ in that location. Obviously, superselection rules do not give any restriction in the manipulation of these states. More specifically, a genuine qubit can be encoded in the subspace spanned by the states $|01\rangle,|10\rangle$, and in this case one can readily verify that all known normal quantum information tasks such as teleportation and quantum error correction can be implemented.

Finally, we discuss a possible set-up where our ideas can be physically implemented and proof of principle experiments may be carried out. We propose to use atoms as particles, since the number of atoms can be considered as a superselection rule. Let us consider, for simplicity, a set of bosonic atoms, each of them with two internal (ground) levels $|a\rangle$ and $|b\rangle$. We will assume that they are at very low temperature, as it is usually achieved in Bose-Einstein condensation experiments 5 . We will also ignore the effect of interactions, something which can be achieved by appropriately tuning the scattering length. We will denote by $a_{0}\left(b_{0}\right)$ the annihilation operators of atoms in internal state $|a\rangle(|b\rangle)$ and motional state $\left|\psi_{0}\right\rangle$, the one corresponding to the ground state of the BoseEinstein condensate. Denoting by $N$ the initial number of atoms, the initial state can be written in second quantization as

$$
|\Psi(0)\rangle=\frac{\left(a_{0}^{\dagger}\right)^{N}}{\sqrt{N !}}|\operatorname{vac}\rangle .
$$

If a pair of laser in Raman configuration is applied for the appropriate time, the state will be the same as before but with $a_{0} \rightarrow\left(a_{0} \pm b_{0}\right) / \sqrt{2}$, where the sign \pm can be easily adjusted with the laser phase-difference and it is chosen according to the value of the bit that needs to be hidden. We will assume that the trap holding the atoms in the internal state $a$ can be manipulated independently of the one for atoms in $b$ 17]. Thus, they can now adiabatically be spatially separated, so that the atoms remaining in state $|a\rangle$ are in a different location than those in $|b\rangle$. The first are given to Alice, where the second are given to Bob. One can readily see that the sign \pm cannot be read by using local measurement and classical communication, something which is due to the conservation of the atom number. In order to read the result, one can bring the atoms back to the initial situation and measure the internal atomic state by applying a laser pulse that transforms $a_{0} \rightarrow\left(a_{0}+b_{0}\right) / \sqrt{2}$ and $b_{0} \rightarrow\left(a_{0}-b_{0}\right) / \sqrt{2}$. If the atoms are found in state $|a\rangle$ the bit was zero and otherwise it was one. Let us emphasize that a Bose-Einstein condensate is not required to perform this experiments, and that even the typical atom interferometry experiments already show the effect that we are discussing [18]. On the other hand, the number of atoms $N$ does not need to be known. Moreover, the readout of the state of the bit can be accomplished by using another Bose-Einstein condensate which is split again into two parts but now with a well defined value of the sign. An interference experiment in each of the sides will then reveal the value of the bit [19]. Finally, let us remark that, in practice, one can also perform the experiments with a simple set-up which uses laser light instead of atoms.

We thank Sandu Popescu for triggering our interest in superselection rules and quantum information. This work was supported in part by the E.C. (RESQ IST2001-37559) and the Kompetenznetzwerk "Quanteninformationsverarbeitung" der Bayerischen Staatsregierung.

[1] C.H. Bennett and G. Brassard, Proc. of the IEEE Int. Conf. on Comp., Systems and Signal Proc., 175 (1984).

[2] W.K. Wootters and W.H. Zurek, Nature 299, 802 (1982).

[3] G.C. Wick, A.S. Wightman and E.P. Wigner, Phys. Rev. 88, 101 (1952).

[4] A. Galindo and P. Pascual, Quantum Mechanics I, Springer-Verlag, Berlin (1990).

[5] E.A. Cornell and C.E. Wieman, Rev. Mod. Phys. 74, 875 (2002); W. Ketterle, Rev. Mod. Phys. 74, 1131 (2002).

[6] J. Walgate et al., Phys. Rev. Lett. 85, 4972 (2000).

[7] B.M. Terhal, D.P. DiVincenzo and D.W. Leung, Phys. Rev. Lett. 86, 5807 (2001); D.P. DiVincenzo et al., IEEE Trans. Inf Theory 48, 580 (2002); D.P. DiVincenzo et al., quant-ph/0207147

[8] T. Eggeling and R.F. Werner, Phys. Rev. Lett. 89, 097905 (2002).

[9] P. Zanardi, Phys. Rev. Lett. 87, 077901 (2001).

[10] H. Barnum et al., quant-ph/0207149 and quant-ph/0305023

[11] H.M. Wiseman and J.A. Vaccaro, quant-ph/0210002 S.D. Bartlett and H.M. Wiseman, quant-ph/0303140

[12] R.F. Werner, Phys. Rev. A 40, 4277 (1989).

[13] H.K. Lo and H.F. Chau, Phys. Rev. Lett. 78, 3410 (1997); D. Mayers, Phys. Rev. Lett. 78, 3414 (1997).

[14] This can be proven as follows: given two pure states for which Bob's reduced density operators are equal, then the two pure states are equal up to a local unitary transformation at Alice's side, even in the presence of superselection rules. Therefore absolute security with relation to cheating for Bob implies absolute freedom to cheating for Alice. See also D. Mayers, quant-ph/0212159

[15] N. Schuch, F. Verstraete and J.I. Cirac, In preparation.

[16] A similar effect applies in the normal quantum data hiding protocols, where it was shown that an amount of entanglement proportional to the dimension of the Hilbert space of the hiding states is necessary to reveal the secret. Note however that the scaling behaviour is much more favorable in the present case, where in principle an infinite amount of entanglement is needed even when the hiding state is a qubit.

[17] D. Jaksch et al., Phys. Rev. Lett. 82, 1975 (1999).

[18] O. Carnal and J. Mlynek, Phys. Rev. Lett. 66, 2689 (1991); D.W. Keith et al., Phys. Rev. Lett. 66, 2693 
(1991).

Castin and J. Dalibard Phys. Rev. A 55, 4330 (1997).

[19] J.I. Cirac et al., Phys. Rev. A 54, R3714 (1996); Y. 